\title{
The air matters - sleeve air cavity as a marker guiding image-guided helical tomotherapy to target cervical cancer
}

\author{
Ya-Ting Jan, MD!, Chih-Long Chang, MD, PhD², Hung-Chi Tai, BS 3 , Yu-Chuen Huang, PhD ${ }^{4,5}$, Chia-Ling Liao, BS ${ }^{3}$, \\ Yu-Jen Chen, MD, PhD ${ }^{3}$ \\ 'Department of Radiology, ${ }^{2}$ Department of Obstetrics and Gynecology, ${ }^{3}$ Department of Radiation Oncology, MacKay Memorial Hospital, \\ Taipei, Taiwan, ${ }^{4}$ Department of Medical Research, China Medial University Hospital, Taichung, Taiwan, ${ }^{5}$ School of Chinese Medicine, \\ College of Chinese Medicine, China Medical University, Taichung, Taiwan
}

\begin{abstract}
Purpose: Radiotherapy with concurrent chemotherapy has been recommended as standard treatment for locally advanced cervical cancer. To validate the main tumor location before each high-precision helical tomotherapy (HT) fraction, the development of a more reliable marker or indicator is of clinical importance to avoid inadequate coverage of the main tumor.

Material and methods: A 61-year-old woman with cervical cancer, TMN stage cT2b2N1M1, FIGO stage IVB was presented. Extended field external beam radiotherapy (EBRT) with concurrent chemotherapy and the interdigitated delivery of intracavitary brachytherapy was performed. Helical tomotherapy equipped with megavoltage cone beam computed tomography (MV-CBCT) was used for image-guided radiotherapy. For the insertion of tandem of brachytherapy applicator, a silicone sleeve with a central hollow canal was placed into the endocervical canal with the caudal end stopping at the outer surface of the cervical os, and making contact with the distal boundary of the cervical tumor during the entire brachytherapy course.

Results: In the remaining EBRT fractions, we found that the air cavity inside the central hollow canal of the sleeve could be clearly identified in daily CBCT images. The radiation oncologists matched the bony markers to adjust the daily setup errors because the megavoltage of the CBCT images could not provide a precise boundary between the soft tissue and the tumor, but the sleeve air cavity, with a clear boundary, could be used as a surrogate and reliable marker to guide the daily setup errors, and to demonstrate the primary tumor location before delivery of each HT fraction.

Conclusions: The application of the sleeve during the interdigitated course of HT and brachytherapy in this patient provided information for the feasibility of using the sleeve air cavity as a surrogate marker for the localization of the main primary tumor before the daily delivery of image-guided HT.
\end{abstract} DOI: $10.5114 /$ jcb.20l6.57803

Key words: brachytherapy, cervical cancer, sleeve air, tomotherapy.

\section{Purpose}

For locally advanced cervical cancer, concurrent chemotherapy and radiation therapy (CCRT) has been recommended as a standard treatment $[1,2]$. However, radiotherapy (RT) complications remain as clinical issues to be improved because of the vicinity of bone marrow and the small bowel, rectum, cervix uteri, and urinary bladder within RT fields [3,4]. Helical tomotherapy (HT), an advanced RT technique providing rotational beam delivery, has been shown to improve target conformality and to spare more normal tissue in comparison with intensitymodulated radiotherapy (IMRT) [5,6].
For the accurate delivery of RT to the target volumes, kilovoltage $(\mathrm{kV})$ cone beam computed tomography (CBCT) scanners were integrated into IMRT machines to perform image-guided radiotherapy (IGRT). The energy of CBCT scanners equipped on HT machines is in megavolts (MV), which does not have the same performance characteristics as diagnostic computed tomography (CT) scanners and $\mathrm{kV}-\mathrm{CBCT}$ because of the lower contrast resolution [7]. The inferior resolution of MV-CBCT images without contrast medium enhancement usually results in an insufficient ability to differentiate tumor and surrounding tissues during the daily verification of patient positioning. The combination of variable factors, including tumor shrink-
Address for correspondence: Yu-Jen Chen, MD, PhD, Department of Radiation Oncology, MacKay Memorial Hospital, 92 Chung San North Road, Section 2, Taipei 104, Taiwan, phone: +886 2 28094661, fax: +886 2 28096180, $\bowtie$ e-mail: chenmdphd@gmail.com
Received: 14.09 .2015

Accepted: 11.01.2016

Published: 26.02.2016 
age, anatomical alteration, body contour change, and daily setup errors, renders the registration of initial planning $\mathrm{CT}$ to $\mathrm{CBCT}$ images difficult. In clinical practice, radiation therapists commonly need to manually adjust the registered images according to bony markers, the most identifiable structure in $\mathrm{CBCT}$, to fit the initial planning target volumes. To validate the main tumor location before each high-precision HT fraction, the development of a more reliable marker or indicator is of clinical importance to avoid inadequate coverage of the main tumor.
Here, we present a case of locally advanced cervical cancer that received HT with the interdigitated combination of intracavitary brachytherapy. For the insertion of tandem of brachytherapy applicator, a silicone sleeve was placed into the endocervix before the beginning of brachytherapy. We found that the air cavity in the sleeve had a clear boundary and could serve as a reliable indicator for locating the main primary tumor during the daily verification of patient positioning in HT practice.
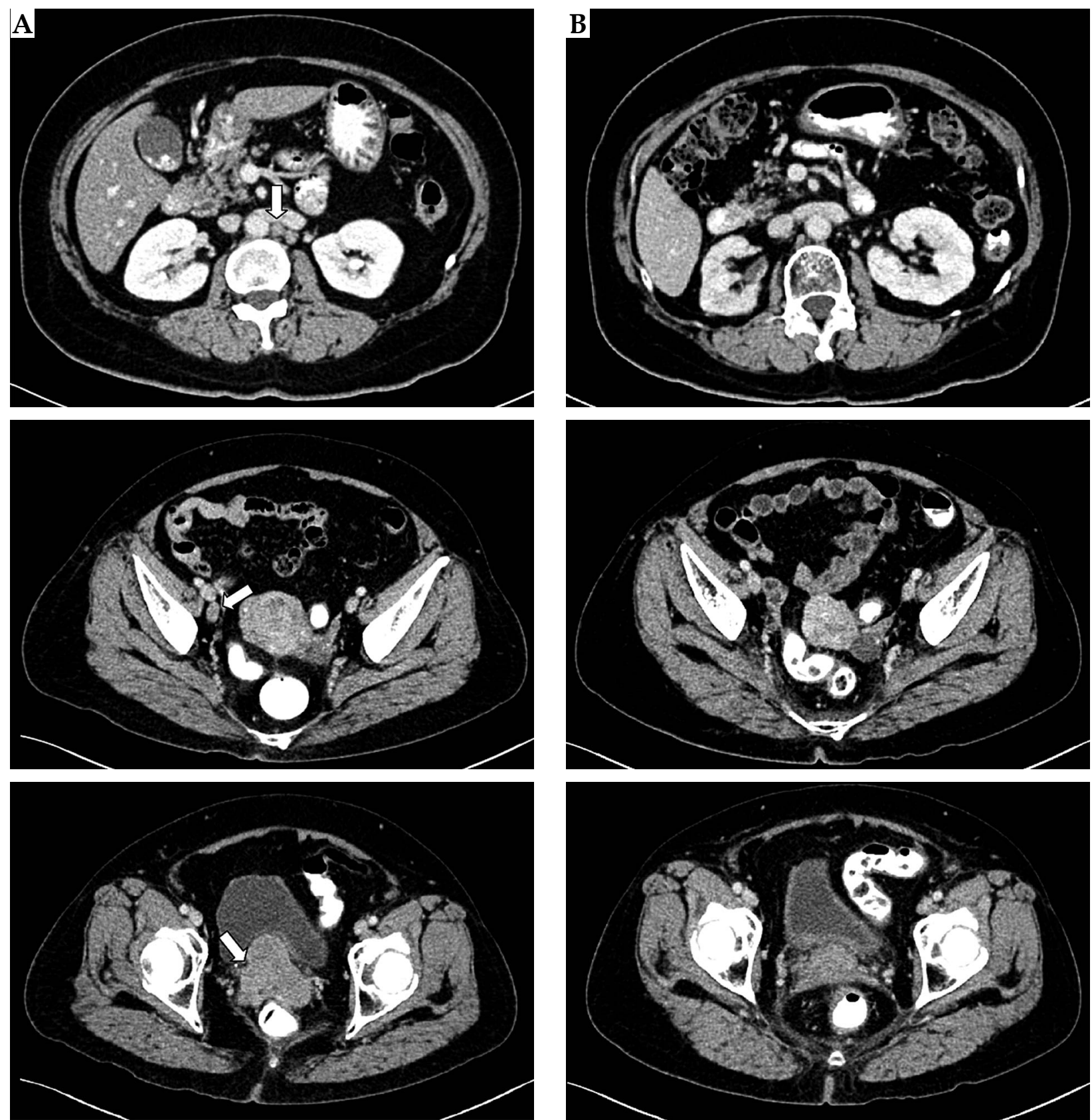

Fig. 1. Diagnostic computed tomography images for primary cervical cancer and lymphadenopathy. A) Before concurrent chemotherapy and radiation therapy (CCRT) treatment, B) after CCRT treatment. Upper panel: renal vein level, arrow indicates enlarged paraaortic lymph node; middle panel: external iliac vein level, arrow indicates enlarged right pelvic lymph node; lower panel: acetabulum level, arrow indicates cervical tumor mass 


\section{Case report}

A 61-year-old woman presented with post-menopause vaginal bleeding was diagnosed as having cervical squamous cell carcinoma by biopsy with parametrial involvement and paraaortic lymphadenopathy noted in CT images (Figure 1A), FIGO stage cT2b2N1M1, IVB. External beam radiotherapy (EBRT) with concurrent chemotherapy, and the interdigitated delivery of intracavitary brachytherapy (high-dose-rate by using ${ }^{192}$ Ir) after
34 Gy of EBRT was performed from December 2015 to January 2016. External beam radiotherapy was planned for $60 \mathrm{~Gy}$ for the main tumor and parametrium as well as 50 Gy to the pelvic and paraaaortic lymph node areas in 30 fractions using the simultaneously integrated boost technique. Helical tomotherapy (Hi Art II with dynamic jaws) in conjunction with MV-CBCT was used to administer EBRT in an image-guided mode. Brachytherapy was designed for $30 \mathrm{~Gy}$ in 6 fractions once a week during the EBRT course and twice a week after the ending of EBRT.
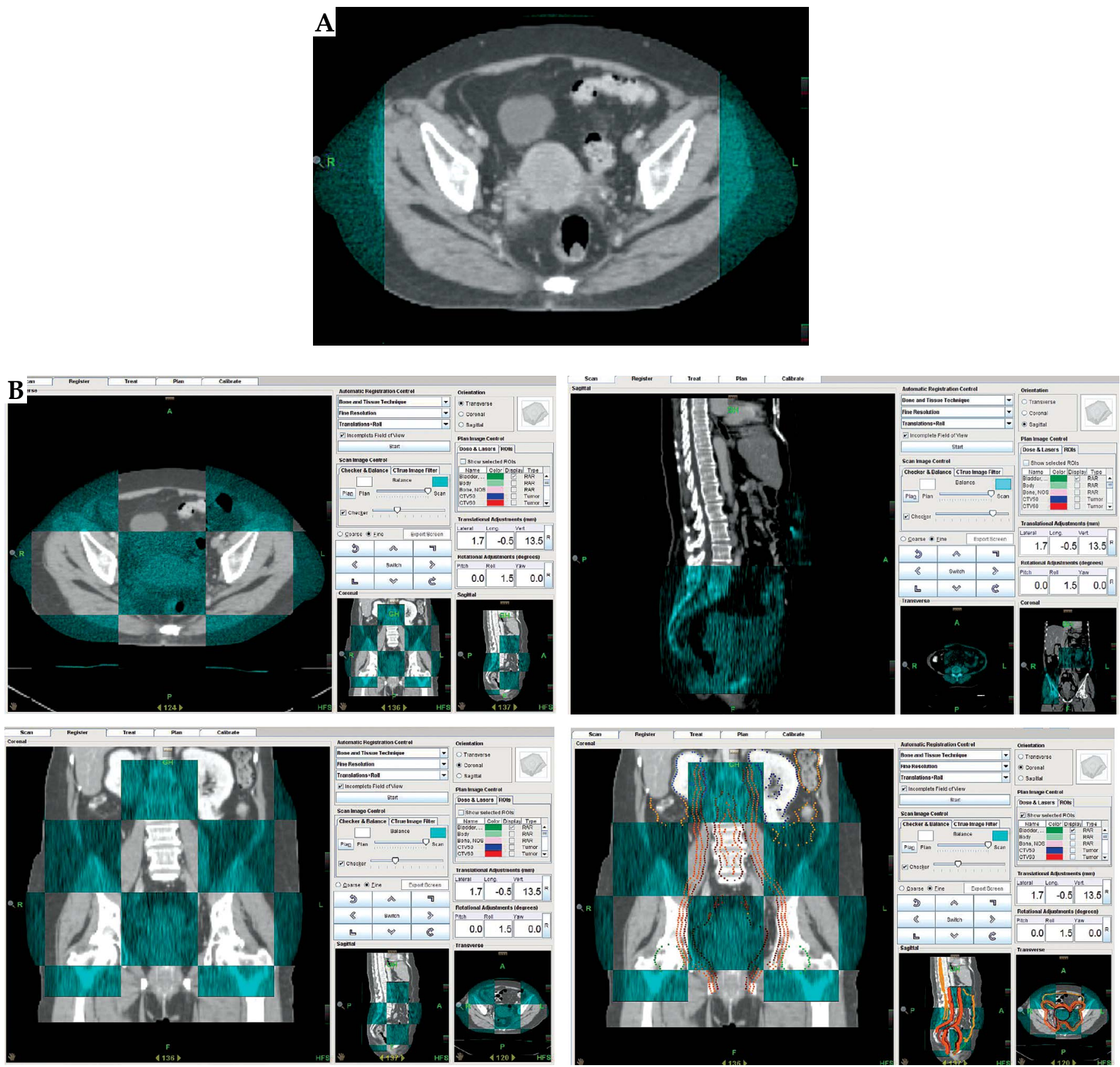

Fig. 2. Image registration of initial planning and cone beam computed tomography (CT). A) Initial planning CT images registered with cone beam CT images. B) Image registration without sleeve insertion. Upper left panel: cross section. Upper right panel: sagittal section. Lower left panel: coronal view. Lower right panel: coronal section with isodose lines 

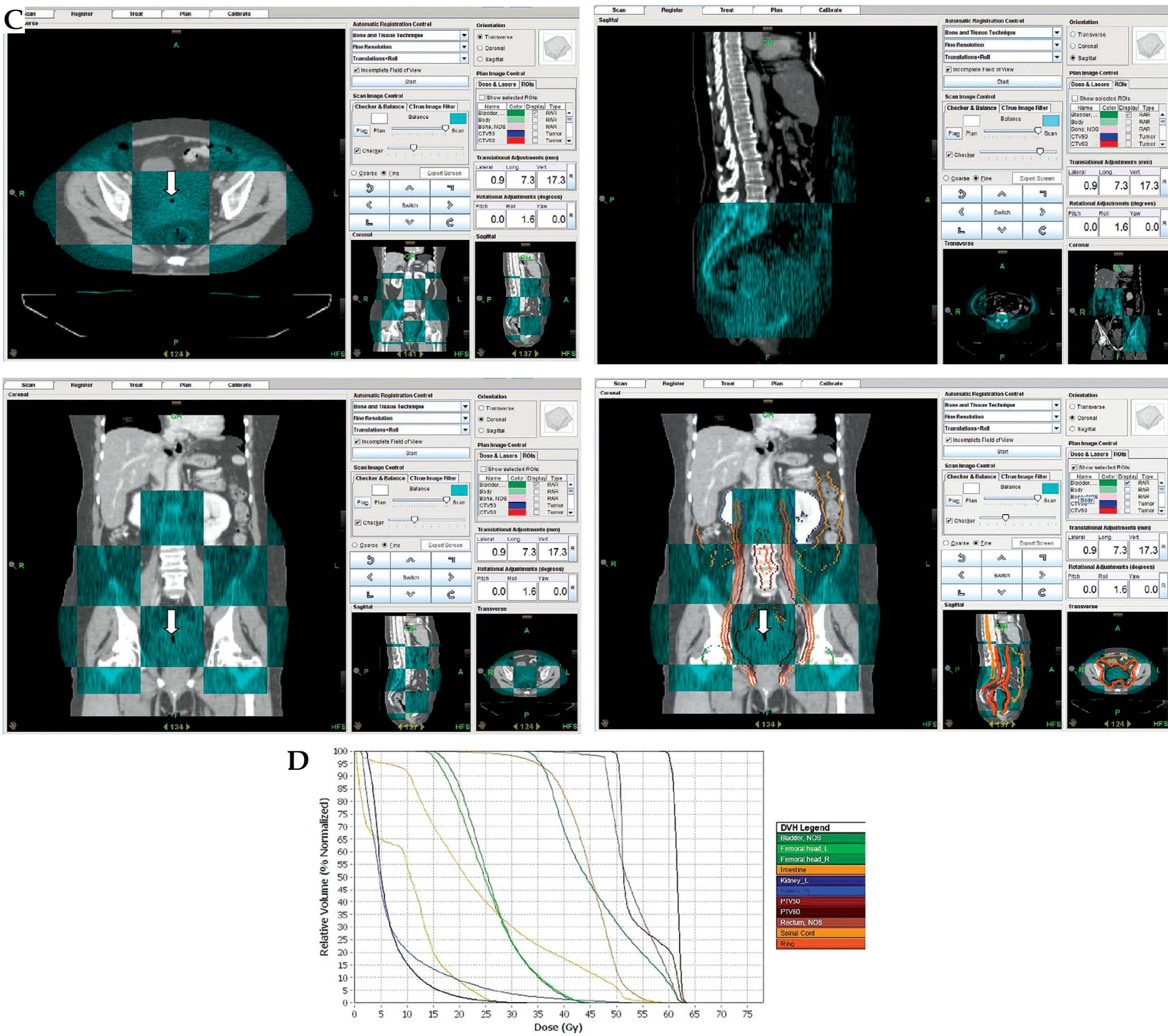

Fig. 2. Cont. Image registration of initial planning and cone beam computed tomography (CT). C) Image registration with sleeve insertion. Upper left panel: cross section. Upper right panel: sagittal section. Lower left panel: coronal view. Lower right panel: coronal section with isodose lines. Grey scale: initial planning CT image; Blue scale: cone beam CT image; Arrow: sleeve air cavity. D) Dose volume histogram of tomotherapy planning

The dose of brachytherapy was set $100 \%$ at point A. For external beam treatment, the dose to $95 \%$ volume of PTV for 50 Gy and 60 Gy was achieved. Mean doses to surrounding normal organs were as follows: 53.0 Gy for rectum, 46.0 Gy for urinary bladder, 24.4 Gy for small bowel, 25.6 Gy for left femoral head, and 26.2 Gy for right femoral head. External beam treatment was planned by using CT-based simulation (Figure 2A) and the dose volume histogram of tomotherapy planning was demonstrated in Figure 2D. For insertion of tandem of brachytherapy applicator, a silicone sleeve (manufactured by Fortune Medical Instrument Corp., New Taipei City, Taiwan) with a central hollow canal (Figure 3A) was used. It was placed into the endocervical canal with the caudal end stopping at the outer surface of the cervical os, and making contact with the distal boundary of the cervical tumor during the entire brachytherapy course. Subsequently, a planned kilovoltage CT scan was performed for visualization and validation of the sleeve insertion (Figure 3B-3D).

\section{Results}

In the remaining EBRT fractions overlapping the brachytherapy course, we found that the air cavity inside the central hollow canal of the sleeve could be clearly identified in the daily MV-CBCT images (Figure 2C). 

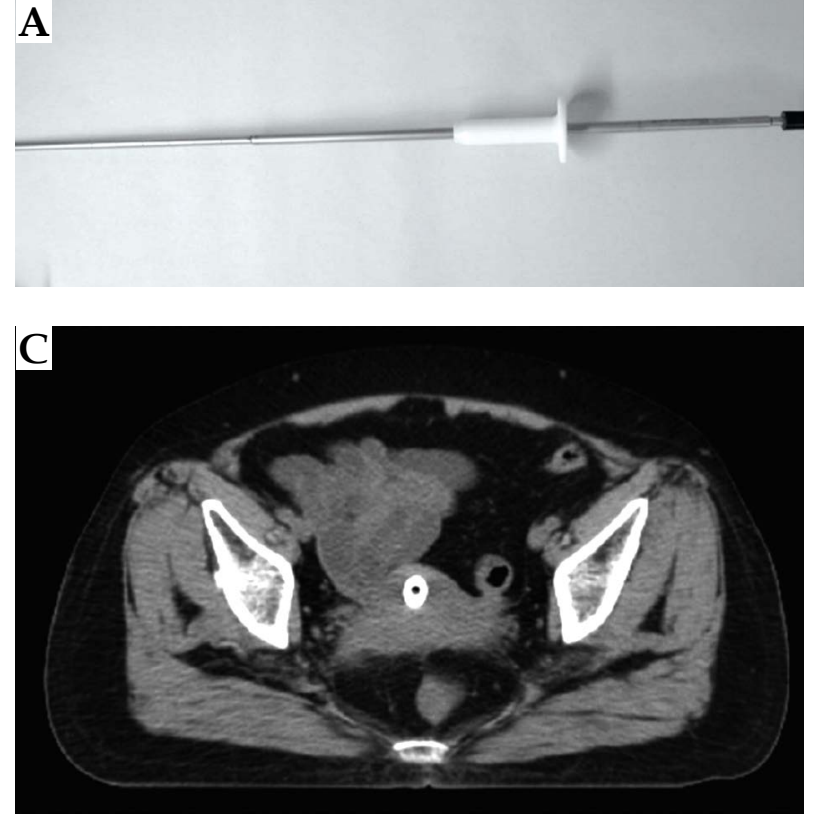

Fig. 3. Demonstration of tandem insertion through sleeve and kilovoltage computed tomography scan images after sleeve insertion. A) White apparatus indicates sleeve, B) oblique coronal view, C) axial view, D) sagittal view

The radiation oncologists matched the bony markers to adjust the daily setup errors because the MV-CBCT images could not provide a precise boundary for the soft tissue or the tumor, only for the bone (Figure 2B). Moreover, the main structure of sleeve was not visible in MV-CBCT images due to lower contrast resolution. The visualization of sleeve by kilovoltage planning CT scan could verify the limitation of MV-CBCT in this scenario. However, the sleeve air cavity had a clear boundary and could be used as a surrogate and reliable marker to guide the daily setup errors, and to demonstrate the primary tumor location before the delivery of each HT fraction. After the completion of the HT and brachytherapy course, the tumor and pelvic and paraaortic lymphadenopathy responded to CCRT (Figure 1B) with acceptable toxicity. No specific
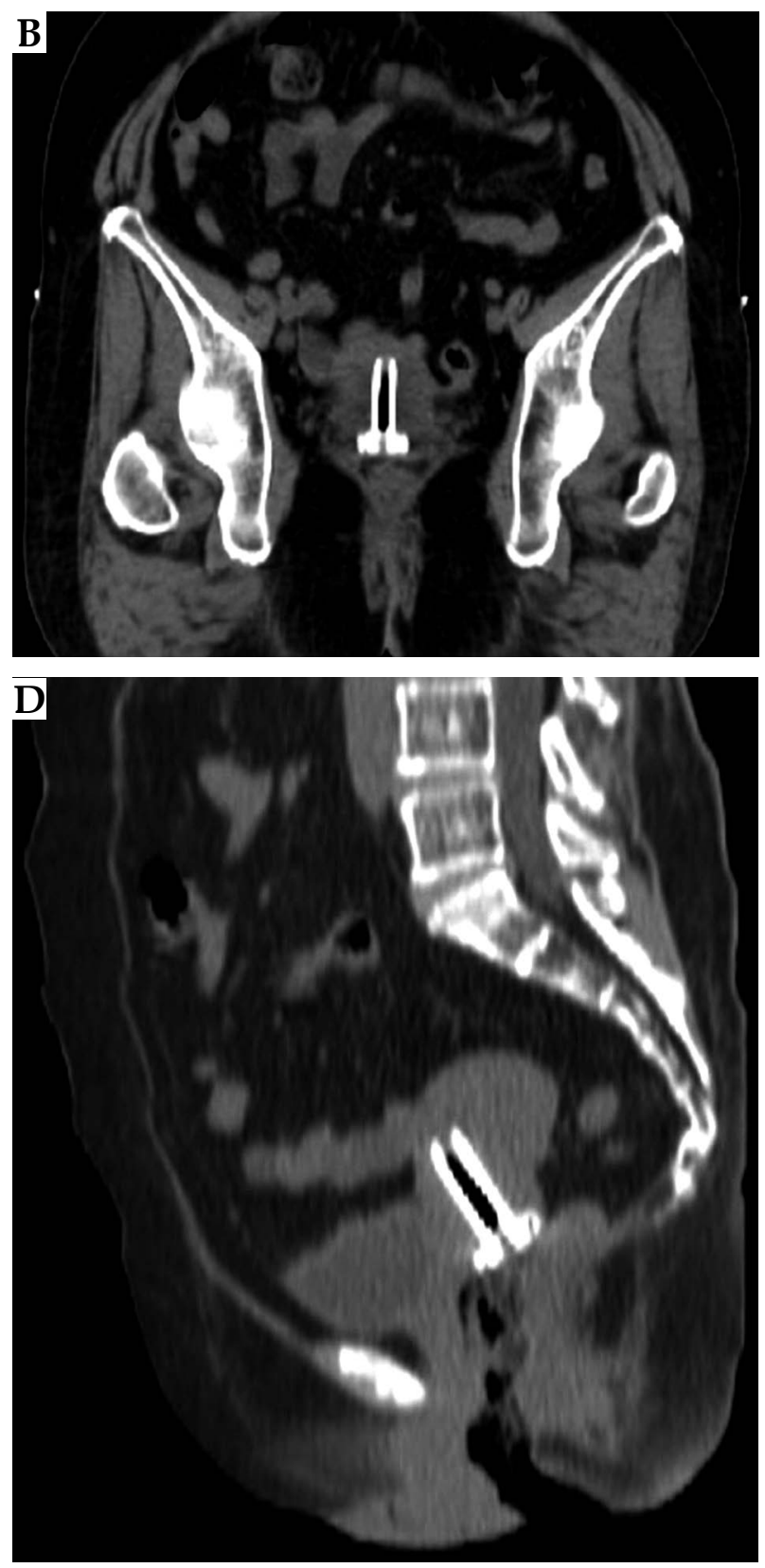

complications were noted related to the insertion of the sleeve for 11 months.

\section{Discussion}

In summary, the application of the sleeve during the interdigitated course of HT and brachytherapy in this patient provided information for the feasibility of using the sleeve air cavity as a surrogate marker for the localization of the main primary tumor before the daily delivery of image-guided HT. This result may suggest the desirability of designing a clinical proposal for early integration of the sleeve during the HT course for a more precise target localization and validation.

The possible limitation of using the sleeve air cavity as an indicator for the main tumor location remains to 
be carefully considered. For example, the insertion of the sleeve early in the EBRT course might be difficult due to the anatomical alteration caused by the bulky tumor at the cervical os $[8,9]$. The relatively long term of insertion of the sleeve may increase the pain scale, vaginal edema, and the risk of infection [10]. The unexpected scattering of the radiation dose from the sleeve needs further dose measurement in the phantom, especially when the scatter correction of CBCT is concerned [11]. Taken together, the early use of the sleeve during the EBRT course, instead of the insertion of the sleeve in a standard interdigitated brachytherapy course, should be performed with caution and could be validated by further clinical trials.

Concerning the possible application of magnetic resonance imaging (MRI) simulation in the future [12,13], the scattering and artifacts surrounding the sleeve might be an issue that should be addressed. To enhance the applicability in both CT- and MRI-based simulations, the modification of the sleeve component could be expedited in collaboration with material scientists [14].

The application of HT in the treatment of cervical cancer is on the increase due to the concerns of normal tissue toxicity and quality of life. Therefore, the efforts to further augment precision in the daily delivery of EBRT by HT is a crucial issue to be addressed. Previous use of metal clips to indicate tumor at our center was not satisfactory, as the clips were not clearly visible on the CBCT images of the HT machine. The adoption of the sleeve air cavity as a marker guiding HT is the first in the literature, so the clinical validation for this issue is therefore warranted. Regardless of using tomotherapy or IMRT, using sleeve may help image guidance during CBCT.

\section{Conclusions}

The application of the sleeve during the interdigitated course of HT and brachytherapy in this patient provided information for the feasibility of using the sleeve air cavity as a surrogate marker for the localization of the main primary tumor before the daily delivery of image-guided HT. Further clinical validation for this issue is however warranted.

\section{Acknowledgments}

This work was supported in part by MMH-E-104-13 from MacKay Memorial Hospital, Taipei, Taiwan.

\section{Disclosure}

Authors report no conflict of interest.

\section{References}

1. Rose PG, Bundy BN, Watkins EB et al. Concurrent cisplatinbased radiotherapy and chemotherapy for locally advanced cervical cancer. N Engl J Med 1999; 340: 1144-1153.

2. Keys HM, Bundy BN, Stehman FB et al. Cisplatin, radiation, and adjuvant hysterectomy compared with radiation and adjuvant hysterectomy for bulky stage IB cervical carcinoma. N Engl J Med 1999; 340: 1154-1161.

3. Mell LK, Tiryaki H, Ahn KH et al. Dosimetric comparison of bone marrow-sparing intensity-modulated radiotherapy versus conventional techniques for treatment of cervical cancer. Int J Radiat Oncol Biol Phys 2008; 71: 1504-1510.

4. Marnitz S, Lukarski D, Köhler C et al. Helical tomotherapy versus conventional intensity-modulated radiation therapy for primary chemoradiation in cervical cancer patients: an intraindividual comparison. Int J Radiat Oncol Biol Phys 2011; 81: 424-430.

5. Kim YB, Kim JH, Jeong KK et al. Dosimetric comparisons of three-dimensional conformal radiotherapy, intensity-modulated radiotherapy, and helical tomotherapy in whole abdominopelvic radiotherapy for gynecologic malignancy. Technol Cancer Res Treat 2009; 8: 369-377.

6. Yang $\mathrm{R}, \mathrm{Xu} \mathrm{S}$, Jiang $\mathrm{W}$ et al. Integral dose in three-dimensional conformal radiotherapy, intensity-modulated radiotherapy and helical tomotherapy. Clin Oncol (R Coll Radiol) 2009; 21: 706-712.

7. Meeks SL, Harmon JF, Jr, Langen KM et al. Performance characterization of megavoltage computed tomography imaging on a helical tomotherapy unit. Med Phys 2005; 32: 2673-2681.

8. Schindel J, Zhang W, Bhatia SK et al. Dosimetric impacts of applicator displacements and applicator reconstruction-uncertainties on 3D image-guided brachytherapy for cervical cancer. J Contemp Brachytherapy 2013; 5: 250-257.

9. Van den Bos W, Beriwal S, Velema L et al. Image guided adaptive brachytherapy for cervical cancer: dose contribution to involved pelvic nodes in two cancer centers. J Contemp Brachytherapy 2014; 6: 21-27.

10. Tyrie LK, Hoskin PJ. Intrauterine high dose rate afterloading brachytherapy: experience of fractionated therapy using a cervical sleeve technique. Clin Oncol (R Coll Radiol) 1996; 8: 376-379.

11. Bootsma GJ, Verhaegen F, Jaffray DA. Efficient scatter distribution estimation and correction in CBCT using concurrent Monte Carlo fitting. Med Phys 2015; 42: 54-68.

12. Chen L, Price RA, Jr., Wang L et al. MRI-based treatment planning for radiotherapy: dosimetric verification for prostate IMRT. Int J Radiat Oncol Biol Phys 2004; 60: 636-647.

13. Arroyo-Cobo JM. A case of self harm in a psychotic patient. Rev Esp Sanid Penit 2015; 17: 33-34.

14. Smit BJ, van Wijk AL. An improved, disposable indwelling intrauterine tube ("smit sleeve") not requiring retaining stitches for brachy-radiotherapy for carcinoma of the cervix. Eur J Gynaecol Oncol 2013; 34: 289-290. 